\title{
Graphene oxide films integrated with nanowires and ring resonators to enhance nonlinear optics
}

This paper was downloaded from TechRxiv (https://www.techrxiv.org).

\section{LICENSE}

CC BY 4.0

SUBMISSION DATE / POSTED DATE

26-01-2022 / 01-02-2022

\section{CITATION}

Moss, David (2022): Graphene oxide films integrated with nanowires and ring resonators to enhance nonlinear optics. TechRxiv. Preprint. https://doi.org/10.36227/techrxiv.19067789.v1

$\mathrm{DOI}$ 


\title{
Graphene oxide films integrated with nanowires and ring resonators to enhance nonlinear optics
}

\author{
Yuning Zhang ${ }^{\mathrm{a}}$, Jiayang $\mathrm{Wu}^{\mathrm{a}}$, Yang $\mathrm{Qu}^{\mathrm{a}}$, Yunyi Yang ${ }^{\mathrm{b}}$, Linnan Jia ${ }^{\mathrm{a}}$, Baohua Jia ${ }^{\mathrm{b}}$, and David J. Moss ${ }^{\mathrm{a}}$ * \\ ${ }^{a}$ Optical Sciences Centre, Swinburne University of Technology, Hawthorn, VIC 3122, Australia \\ ${ }^{\mathrm{b}}$ Centre for Translational Atomaterials, Swinburne University of Technology, Hawthorn, VIC 3122, Australia \\ * Electronic mail: dmoss@swin.edu.au
}

\begin{abstract}
We report enhanced nonlinear optics in nanowires, waveguides, and ring resonators by introducing layered two-dimensional (2D) graphene oxide (GO) films through experimental demonstration. The GO films are integrated on silicon-on-insulator nanowires (SOI), high index doped silica glass, and silicon nitride ( $\mathrm{SiN}$ ) waveguides and microring resonators (MRRs), to demonstrate an improved optical nonlinearity including Kerr nonlinearity and four-wave mixing (FWM). By using a largearea, transfer-free, layer-by-layer GO coating method with photolithography and lift-off processes, we integrate GO films on these complementary metal-oxide-semiconductor (CMOS)-compatible devices. For SOI nanowires, significant spectral broadening of optical pulses in GO-coated SOI nanowires induced by self-phase modulation (SPM) is observed, achieving a high spectral broadening factor of 4.34 for a device with a patterned film including 10 layers of GO. A significant enhancement in the nonlinear figure of merit (FOM) for silicon nanowires by a factor of 20 is also achieved, resulting in a FOM > 5. For Hydex and SiN waveguides, enhanced FWM in the GO-coated waveguides is achieved, where conversion efficiency (CE) enhancements of up to $6.9 \mathrm{~dB}$ and $9.1 \mathrm{~dB}$ relative to the uncoated waveguides. For MRRs, an increase of up to $\sim 10.3 \mathrm{~dB}$ in the FWM CE is achieved due to the resonant enhancement effect. These results reveal the strong potential of GO films to improve the nonlinear optics of nanowires, waveguides, and ring resonators.
\end{abstract}

Keywords: nonlinear optics, CMOS compatible photonic platforms, graphene oxide, Kerr nonlinearity, four-wave mixing

\section{INTRODUCTION}

The $3^{\text {rd }}$ order nonlinear optical response has found wide ranging applications in telecommunications, metrology, astronomy, ultrafast optics, quantum photonics, and many other areas [1-4]. Nonlinear integrated photonic devices based on the Kerr effect $\left(n_{2}\right)$ in particular offer far superior processing speeds compared to electronic devices as well as the added benefits of compact footprint, low power consumption, high stability, and low-cost mass production, all of which are important for highspeed signal generation and processing in optical communication systems [5-7].

While silicon-on-insulator nanowires (SOI) has shown itself to be a leading platform for integrated photonic devices, it suffers from strong two-photon absorption (TPA) at near-infrared wavelengths, which greatly limits the nonlinear performance $[5,6]$, and this has motivated the use of highly nonlinear materials on chips. Other complementary metal-oxidesemiconductor (CMOS) compatible platforms including high index doped silica glass [8,9] and silicon nitride (SiN) [10, 11] have a much lower TPA, but they hamper the nonlinear performance due to a comparatively low Kerr nonlinearity. To overcome these limitations, two-dimensional (2D) layered graphene oxide (GO) has received much attention among the various 2D materials due to its ease of preparation as well as the tunability of its material properties [12-20]. Previously, we reported GO films with a giant Kerr nonlinear response about 4-5 orders of magnitude higher than that of silicon and $\mathrm{SiN}$ [16] and demonstrated enhanced four-wave mixing (FWM) in doped silica waveguides and microring resonators (MRRs) integrated with GO films [21, 22]. Here, we demonstrate enhanced nonlinear optics in SOI nanowires [23] and SiN waveguides [24] integrated with 2D layered GO films. Owing to the strong light-matter interaction between the integrated waveguides and the highly nonlinear GO films, self-phase modulation (SPM) measurements are performed to show significant spectral broadening enhancement for SOI nanowires coated with patterned films of GO. The dependence of GO's Kerr nonlinearity on layer number and pulse energy shows interesting physical insights and trends of the layered GO films in 
evolving from 2D monolayers to quasi bulk-like behavior. We obtain significant enhanced nonlinear performance for the GO hybrid devices as compared with the bare waveguides, achieving the nonlinear parameter of GO-coated SOI nanowires up by 16 times, with the nonlinear figure of merit (FOM) increasing over 20 times to FOM $>5$. We obtain a significant improvement in the FWM conversion efficiency $(\mathrm{CE})$ of $\approx 6.9 \mathrm{~dB}$ for a uniformly coated Hydex waveguide with 2 layer of $\mathrm{GO}$ and $\approx 9.1 \mathrm{~dB}$ for a patterned $\mathrm{SiN}$ waveguide with 5 layers of GO. For MRRs, we achieve up to $\sim 10.3 \mathrm{~dB}$ in the FWM CE. These results confirm the strong potential of introducing 2D layered GO films into CMOS compatible photonic platforms to realize high-performance nonlinear photonic devices.

\section{ENHANCED KERR NONLINEARITY IN GO-COATED SILICON-ON-INSULATOR NANOWIRES}

Figure 1a shows a schematic of an SOI nanowire waveguide integrated with a GO film. The fabrication of the SOI nanowire can be achieved via either deep ultraviolet photolithography or e-beam lithography followed by inductively coupled plasma etching, all of which are mature silicon device fabrication technologies $[25,26]$. The GO film coating, with a thickness of $\sim 2$ $\mathrm{nm}$ per layer [23], can be achieved using solution-based methods that yield layer-by-layer film deposition [20, 21, 27]. As compared with the sophisticated transfer processes for other 2D materials such as graphene and TMDCs [28, 29], these coating methods enable transfer-free and conformal film coating, with high fabrication stability, repeatability, precise control of the film thickness (i.e., number of layers), and extremely good film attachment onto integrated photonic devices [30]. Figure $1 \mathrm{~b}$ shows a microscope image of a fabricated SOI chip with a 0.4-mm-long opened window. Apart from allowing precise control of the placement and coating length of the GO films that are in contact with the SOI nanowires, the opened windows also enabled us to test the performance of devices having a shorter length of GO film but with higher film thicknesses (up to 20 layers). This provided more flexibility to optimize the device performance with respect to SPM spectral broadening. Figure 1c shows the scanning electron microscopy (SEM) image of an SOI nanowire conformally coated with 1 layer of GO. Note that the conformal coating (with the GO film coated on both the top surface and sidewalls of the nanowire) is slightly different to earlier work where we reported doped silica devices with GO films only coated on the top surface of the waveguides $[30,31]$. As compared with doped silica waveguides, the SOI nanowires allow much stronger light-material interaction between the evanescent field leaking from the waveguide and the GO film, which is critical to enhance nonlinear optical processes such as SPM and FWM. Figure 1d shows the successful integration of GO films which is confirmed by the representative D (1345 cm-1) and G (1590 cm-1) peaks of GO observed in the Raman spectrum of an SOI chip coated with 5 layers of GO. Microscope images of the same SOI chip before and after GO coating are shown in the insets, which illustrate good morphology of the films.
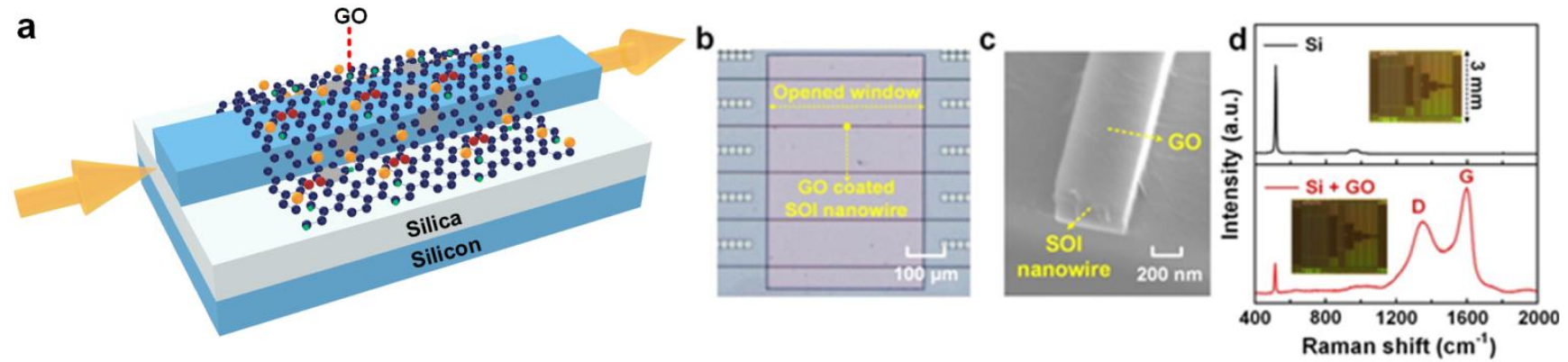

Figure 1. (a) Schematic illustration of a GO-coated SOI nanowire waveguide. (b) Microscope image of a fabricated SOI chip with a 0.4mm-long opened window. (c) Scanning electron microscopy (SEM) image of a SOI nanowire conformally coated with 1 layer of GO. (d) Raman spectra of an SOI chip without GO and with 5 layers of GO. Insets show the corresponding microscope images.

Figure 2 shows the results of the SPM experiments. Figure 2a-i shows the normalized spectra of the optical pulses before and after transmission through the SOI nanowires with 2.2-mm-long, 1-3 layers of GO, together with the output optical spectrum for the bare SOI nanowire, all taken with the same pulse energy of $\sim 51.5 \mathrm{pJ}$ (i.e., $\sim 13.2 \mathrm{~W}$ peak power, excluding coupling loss) coupled into the SOI nanowires. As compared with the input pulse spectrum, the output spectrum after transmission through the bare SOI nanowire exhibited measurable spectral broadening. This is expected and can be attributed to the high Kerr nonlinearity of silicon. The GO-coated SOI nanowires, on the other hand, show much more significantly 
broadened spectra as compared with the bare SOI nanowire, clearly reflecting the improved Kerr nonlinearity of the hybrid waveguides. Figure 2a-ii shows the corresponding results for the SOI nanowires with 0.4-mm-long, 5-20 layers of GO, taken with the same coupled pulse energy as in Figure 2a-i. The SOI nanowires with a shorter GO coating length but higher film thicknesses also clearly show more significant spectral broadening as compared with the bare SOI nanowire. We also note that in either Figure 2a-i or 2a-ii, the maximum spectral broadening is achieved for a device with an intermediate number of GO layers (i.e., 2 and 10 layers of GO in a-i and a-ii, respectively). This could reflect the trade-off between the Kerr nonlinearity enhancement (which dominates for the device with a relatively short GO coating length) and loss increase (which dominates for the device with a relatively long GO coating length) for the SOI nanowires with different numbers of GO layers.

Figures $2 b-i$ and b-ii show the power-dependent output spectra after going through the SOI nanowires with (i) 2 layers and (ii) 10 layers of GO films. We measured the output spectra at 10 different coupled pulse energies ranging from $\sim 8.2 \mathrm{pJ}$ to $\sim 51.5 \mathrm{pJ}$ (i.e., coupled peak power from $\sim 2.1 \mathrm{~W}$ to $\sim 13.2 \mathrm{~W}$ ). As the coupled pulse energy was increased, the output spectra showed increasing spectral broadening as expected. We also note that the broadened spectra exhibited a slight asymmetry. This was a combined result of both the asymmetry of the input pulse spectrum and the free-carrier effect of silicon including both the free carrier absorption (FCA) and free carrier dispersion (FCD). Since the time response for the generation of free carries is slower compared to the pulse width, there is a delayed impact of FCA on the pulse shape, which leads the spectral asymmetry of the optical pulses. The FCD further broadens the asymmetry induced by FCA, resulting in more obvious spectral asymmetry at the output.
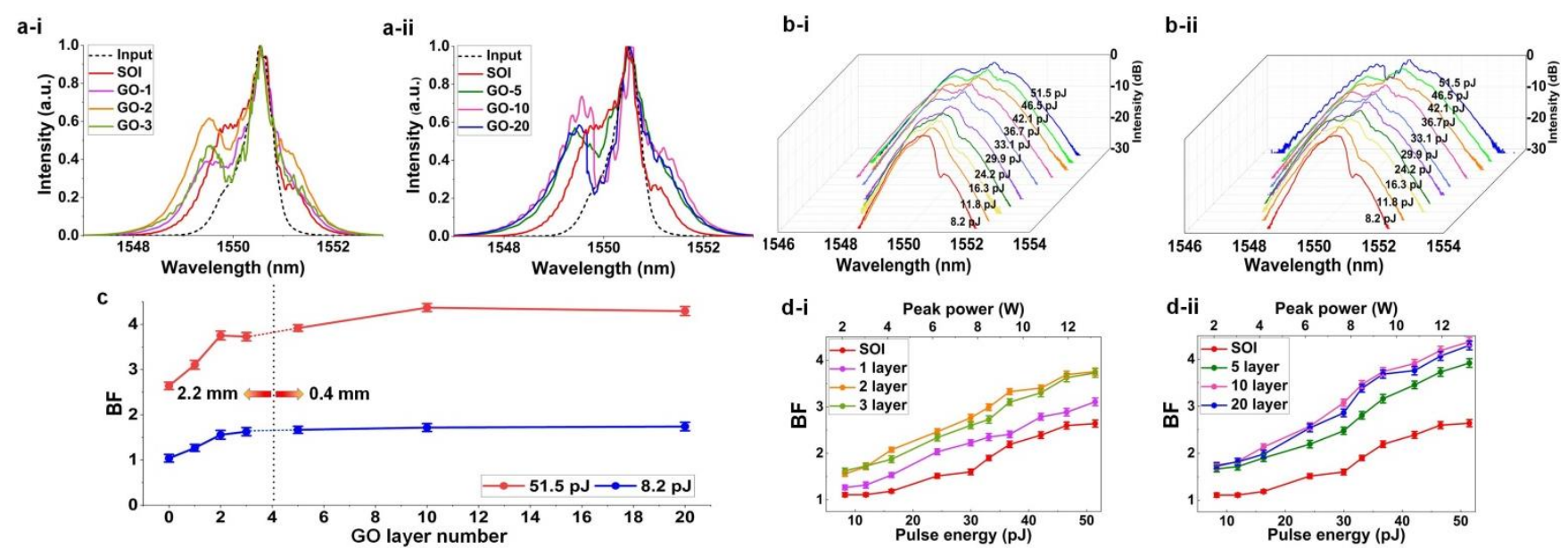

Figure 2. SPM experimental results. (a) Normalized spectra of optical pulses before and after going through the GO-coated SOI nanowires at a coupled pulse energy of $\sim 51.5 \mathrm{pJ}$. (b) Optical spectra measured at different pulse energies for the GO-coated SOI nanowires. (c) BFs of the measured output spectra versus GO layer number at fixed coupled pulse energies of $8.2 \mathrm{pJ}$ and $51.5 \mathrm{pJ}$. (d) BFs of the measured output spectra versus coupled pulse energy (or coupled peak power). In (a), (b) and (d), (i) and (ii) show the results for the SOI nanowires with 2.2-mm-long, 1-3 layers of GO and with 0.4-mm-long, 5-20 layers of GO, respectively. In (a), (c) and (d), the corresponding results for the bare SOI nanowires are also shown for comparison.

To quantitively analyze the spectral broadening of the output spectra, we introduce the concept of a broadening factor (BF, defined as the square of the pulse'rms spectral width at the waveguide output facet divided by the corresponding value at the input [31]). Figure 2c shows the BFs of the measured output spectra after transmission through the bare SOI nanowire and the GO-coated SOI nanowires at pulse energies of $8.2 \mathrm{pJ}$ and $51.5 \mathrm{pJ}$. The GO-coated SOI nanowires show higher BFs than the bare SOI nanowires (i.e., GO layer number $=0$ ), and the BFs at a coupled pulse energy of $51.5 \mathrm{pJ}$ are higher than those at $8.2 \mathrm{pJ}$, agreeing with the results in Figures $2 \mathrm{a}$ and 2b, respectively. At $51.5 \mathrm{pJ}$, BFs of up to 3.75 and 4.34 are achieved for the SOI nanowires with 2 and 10 layers of GO, respectively. This also agrees with the results in Figure 2a - with the maximum spectral broadening being achieved for an intermediate number of GO layers due to the trade-off between the Kerr nonlinearity enhancement and increase in loss. The BFs of the output spectra versus coupled pulse energy are shown in Figures 2d-i and 2d-ii for the SOI nanowires with 1-3 layers and 5-20 layers of GO, respectively. The BFs increase with coupled pulse energy, reflecting a more significant spectral broadening that agrees with the results in Figure $2 \mathrm{~b}$. 


\section{ENHANCED FWM IN GO-COATED HYDEX AND SIN WAVEGUIDES}

Figure 3a shows the GO-coated integrated waveguides made from high-index doped silica glass [5], with a cross section of 2 $\mu \mathrm{m} \times 1.5 \mu \mathrm{m}$. The integrated waveguide is surrounded by silica except that the upper cladding is removed to enable coating the waveguide with GO films. The GO films, with a thickness of about $2 \mathrm{~nm}$ per layer, were introduced on the top of the integrated waveguide in order to introduce light-material interaction with the evanescent field leaking from the integrated waveguide. The Kerr coefficient of GO is on the order of $10^{-15} \sim 10^{-14} \mathrm{~m}^{2} / \mathrm{W}[16,19]$, which is slightly lower than that of graphene $\left(\sim 10^{-13} \mathrm{~m}^{2} / \mathrm{W}\right)[19,32,33]$, but still orders of magnitude higher than that of high-index doped silica glass $\left(\sim 10^{-19}\right.$ $\left.\mathrm{m}^{2} / \mathrm{W}\right)$ and silica $\left(\sim 10^{-20} \mathrm{~m}^{2} / \mathrm{W}\right)$ [5].The waveguides were fabricated via CMOS compatible processes [34, 35]. First, highindex doped silica glass films $(n=\sim 1.60$ at $1550 \mathrm{~nm})$ were deposited using standard plasma enhanced chemical vapour deposition (PECVD), then patterned using deep UV photo-lithography and etched via reactive ion etching (RIE) to form waveguides with exceptionally low surface roughness. After that, silica glass $(n=\sim 1.44$ at $1550 \mathrm{~nm})$ was deposited via PECVD and the upper cladding of the integrated waveguides was removed by chemical mechanical polishing (CMP). Finally, the GO film was coated on the top surface of the chip by a solution-based method that yields layer-by-layer deposition of GO films, as reported previously[20]. An image of the integrated waveguide incorporating two layers of GO is shown in Figure 3b, which illustrates that the morphology is good, leading to a high transmittance of the GO film on top of the integrated waveguide. The integration of GO onto the waveguide is confirmed by Raman spectroscopic measurements (Figure 3c) that show the representative $\mathrm{D}\left(1345 \mathrm{~cm}^{-1}\right)$ and $\mathrm{G}\left(1590 \mathrm{~cm}^{-1}\right)$ peaks of GO [16].

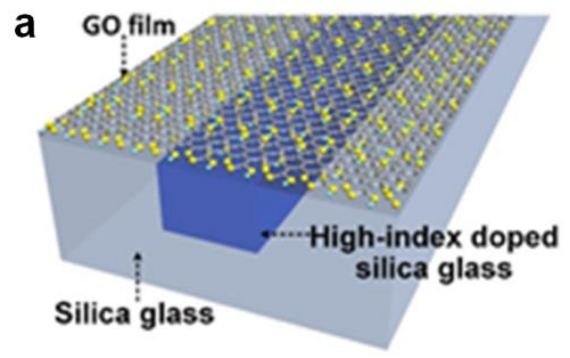

d

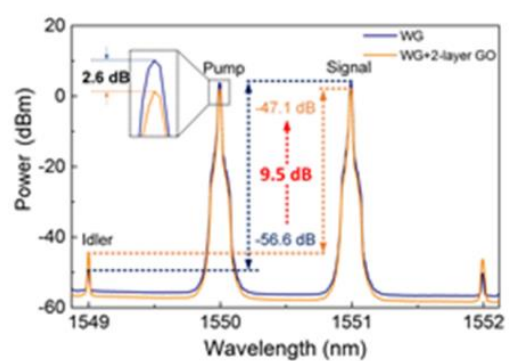

b

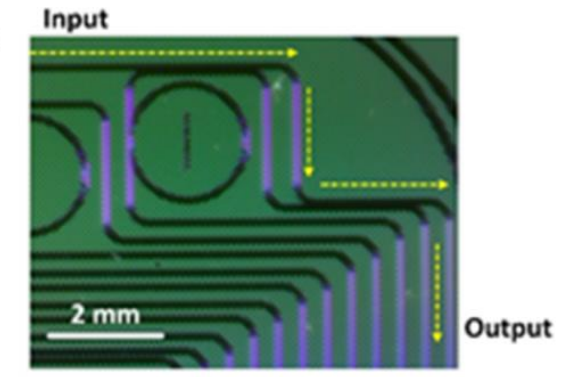

e

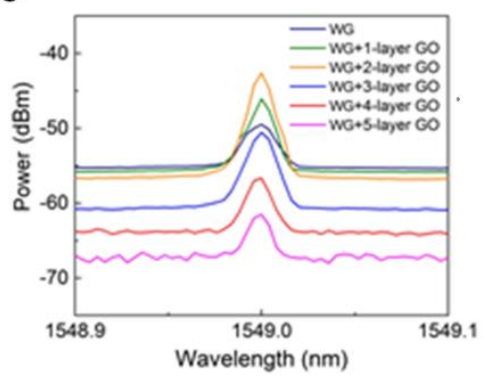

C

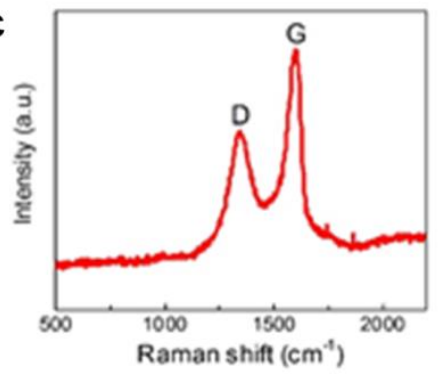

f

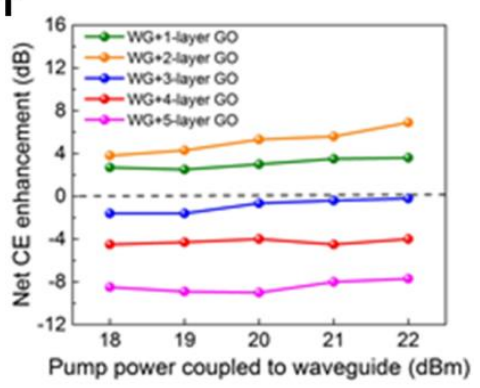

Figure 3. (a) Schematic illustration of hybrid Hydex waveguides integrated with GO. (b) Image of the hybrid Hydex integrated waveguide with GO. (c) Raman spectra of GO on the integrated chip. (d) FWM spectra of the integrated waveguide without GO and with 2 layers of GO. (e) Zoom in spectra of the generated idlers after FWM in the waveguide with 0 to 5 layers of GO. (f) Net CE enhancements for various pump powers coupled to the waveguide with 1 to 5 layers of GO.

The FWM spectra of a 1.5-cm-long integrated waveguide without GO and with 2 layers of GO are shown in Figure 3d. For comparison, we kept the same pump power of $\sim 30 \mathrm{dBm}$ before the input of the waveguide, which corresponded to $\sim 22$ $\mathrm{dBm}$ pump power coupled into the waveguide. It can be seen that although the hybrid integrated waveguide had additional propagation loss $(\sim 2.6 \mathrm{~dB})$, it clearly shows enhanced idler output powers as compared with the same waveguide without GO. The CE (defined as the ratio of the output power of the idler to the output power of the signal, i.e., $\mathrm{P}_{\text {out, idler }} / \mathrm{P}_{\text {out, signal }}$ ) of the integrated waveguide with and without $\mathrm{GO}$ were $\sim-47.1 \mathrm{~dB}$ and $\sim 56.6 \mathrm{~dB}$, respectively, corresponding to a CE enhancement of $\sim 9.5 \mathrm{~dB}$ for the hybrid integrated waveguide. After excluding the addtional propagation loss, the net CE enhancement (defined as the improvement of the output power of the idler for the same pump power coupled to the waveguide) is $6.9 \mathrm{~dB}$. 
For the integrated waveguide coated with 1 to 5 layers of GO, zoom-in spectra of the generated idlers for the same pump power coupled to the waveguide $(\sim 22 \mathrm{dBm})$ are shown in Figure 3e. For the integrated waveguide coated with 1 and 2 layers of GO, there were positive net CE enhancements. When the number of GO layers was over 2, the net change in CE was negative. This is mainly due to the super-linear increase in propagation loss for increased numbers of GO layers as noted above. The output powers of the idler for various pump powers coupled to the waveguide without GO and with 2 layers of GO are shown in Figure 3f.
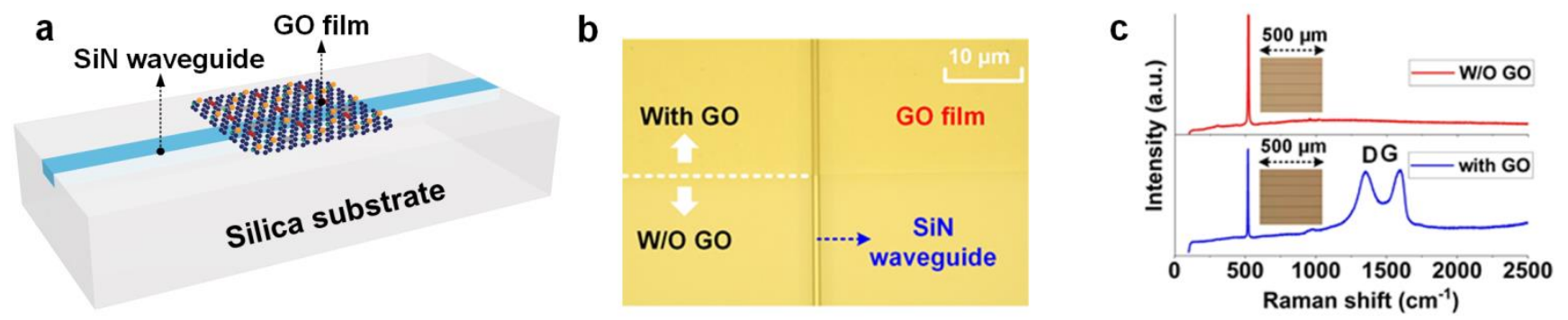

Figure 4. (a) Schematic illustration of GO-coated SiN waveguide. (b) Microscope image of a SiN waveguide patterned with 10 layers of GO. (c) Raman spectra of a SiN chip without GO and with 10 layers of GO. Insets show the corresponding microscope images.

Figure $4 \mathrm{a}$ shows the $\mathrm{SiN}$ waveguide integrated with a GO film. SiN waveguides with a cross section of $1.6 \mu \mathrm{m} \times 0.66 \mu \mathrm{m}$ were fabricated via annealing-free and crack-free processes that are compatible with CMOS fabrication [36, 37]. First, a SiN layer was deposited via low-pressure chemical vapor deposition (LPCVD) in two steps, with a 370-nm-thick layer for each, so as to control strain and to prevent cracks. In order to produce high-quality films, a tailored ultra-low deposition rate $(<2$ $\mathrm{nm} / \mathrm{min}$ ) was used. Waveguides were then formed via a combination of deep ultraviolet lithography and fluorine-based dry etching that yielded exceptionally low surface roughness. Next, a 3- $\mu \mathrm{m}$ thick silica upper cladding layer was deposited via high-density plasma-enhanced chemical vapor deposition (HDP-PECVD) to avoid void formation. To enable the interaction between the GO films and the evanescent field leaking from the SiN waveguides, the silica upper cladding was removed using a perfectly selective CMP that left the top surface of the SiN waveguides exposed in air, with no SiN consumption and no remaining topography. Figure $4 \mathrm{~b}$ shows a microscope image of a SiN waveguide patterned with 10 layers of GO, which illustrates the high transmittance and good morphology of the GO films. Figure 4b presents a scanning electron microscopy (SEM) image of a GO film with up to 5 layers of GO monolayers, clearly showing the layered film structure. Figure 4c shows the measured Raman spectra of a SiN chip without GO and with 10 layers of uniformly coated GO films. The successful integration of GO films is confirmed by the presence of the representative D (1345 cm-1) and G (1590 cm-1) peaks of GO.

Figure 5 shows the experimental FWM optical spectra for the SiN waveguides uniformly coated with 1 and 2 layers of GO (Figure 5a-i) together with the FWM spectrum of the bare SiN waveguide. For comparison, we kept the same power of 23 $\mathrm{dBm}$ for both the pump and signal before the input of the waveguides, which corresponded to $18 \mathrm{dBm}$ power for each coupled into the waveguides. The difference among the baselines of the spectra reflects the difference in waveguide propagation loss for different samples. It can be seen that although the hybrid waveguide with 1 layer of GO film had an additional propagation loss of $\approx 7.1 \mathrm{~dB}$, it clearly shows enhanced idler output powers as compared with the bare SiN waveguide. The $\mathrm{CE}$ of the $\mathrm{SiN}$ waveguides without $\mathrm{GO}$ and with 1 layer of $\mathrm{GO}$ were $\approx-65.7 \mathrm{~dB}$ and $\approx-58.4 \mathrm{~dB}$, respectively, corresponding to a $\mathrm{CE}$ enhancement of $\approx 7.3 \mathrm{~dB}$ for the hybrid waveguide. In contrast to the positive $\mathrm{CE}$ enhancement for the hybrid waveguide with 1 layer of GO, the change in $\mathrm{CE}$ for the hybrid waveguide with 2 layers of GO was negative.

Figure 5a-ii shows the FWM spectra of the SiN waveguides with 5 and 10 layers of patterned GO films. The coupled CW pump and signal power (18 dBm for each) was the same as that in Figure 5a-i. The SiN waveguides with patterned GO films also had an additional insertion loss as compared with the bare waveguide, while the results for both 5 and 10 GO layers show enhanced idler output powers. In particular, there is a maximum $\mathrm{CE}$ enhancement of $\approx 9.1 \mathrm{~dB}$ for the SiN waveguide patterned with 5 layers of GO, which is even higher than that for the uniformly coated waveguide with 1 layer of GO. This reflects the trade-off between FWM enhancement (which dominates for the patterned devices with a short GO coating length) and loss (which dominates for the uniformly coated waveguides with a much longer GO coating length) in the GO-coated $\mathrm{SiN}$ waveguides. Figure $5 \mathrm{~b}$ shows the measured $\mathrm{CE}$ versus pump power for the uniformly coated and patterned devices, 
respectively. The plots show the average of three measurements on the same samples and the error bars reflect the variations, showing that the measured CE is repeatable. As the pump power was increased, the measured CE increased linearly with no obvious saturation for the bare SiN waveguide and all the hybrid waveguides, indicating the low TPA of both the SiN waveguides and the GO films. For the bare waveguide, the dependence of CE versus pump power shows a nearly linear relationship, with a slope rate of about 2 for the curve as expected from classical FWM theory [38]. For the GO-coated waveguides, the measured CE curves have shown slight deviations from the linear relationship with a slope rate of 2 , particularly at high light powers. Figure $5 \mathrm{c}$ compares the $\mathrm{CE}$ of the hybrid waveguides with four different numbers of GO layers (i.e., 1, 2, 5, 10), where we see that the hybrid waveguide with an intermediate number of GO layers has the maximum CE. This reflects the trade-off between $\gamma$ and loss in the hybrid waveguides, which both increase with GO layer number.
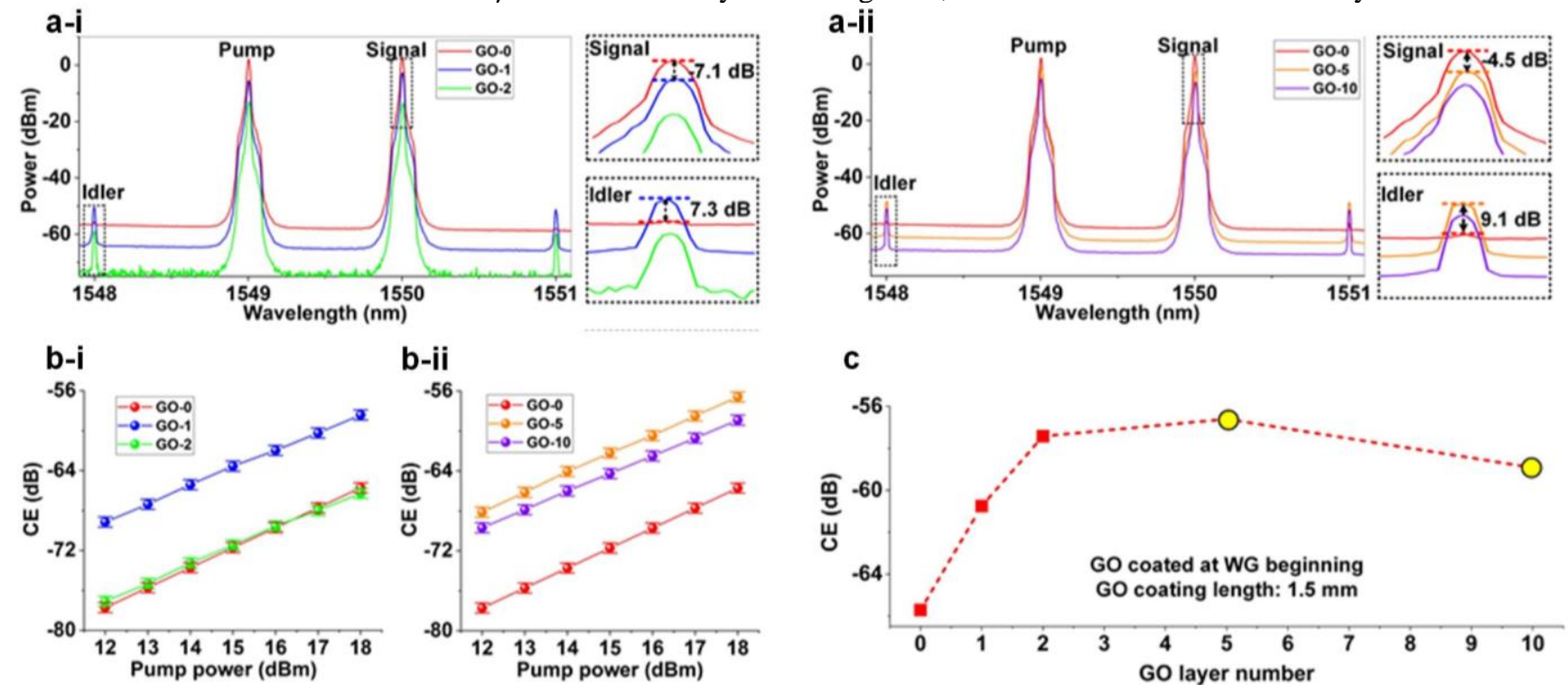

Figure 5. FWM experimental results. (a) FWM optical spectra. Insets show zoom-in view around the signal and idler. (b) CE versus pump power coupled into the waveguides. In (a) and (b), (i) shows the results for SiN waveguides uniformly coated with 1 and 2 layers of GO and (ii) shows the results for SiN waveguides patterned with 5 and 10 layers of GO. (c) Calculated CE as functions of GO layer number. In (c), the corresponding results for the bare $\mathrm{SiN}$ waveguide (GO-0) are also shown for comparison. The coating length is $1.5 \mathrm{~mm}$ and the $\mathrm{GO}$ coating position is at waveguide beginning.

\section{ENHANCED FWM IN GO-COATED MICRO-RING RESONATORS}

Figure 6a shows a schematic of an integrated MRR incorporating a GO film. The MRR was fabricated on a high index doped silica glass platform using CMOS compatible fabrication processes $[8,39]$ with CMP used as the last step to remove the upper cladding, so as to enable GO film coating on the top surface of the MRR. Benefiting from extraordinarily low linear and nonlinear loss, high index doped silica glass has been a successful integrated platform for nonlinear photonic devices [9, 40, 41]. The Kerr coefficient $n_{2}$ of the high index doped silica glass $\left(\sim 1.3 \times 10^{-19} \mathrm{~m}^{2} / \mathrm{W}\right)$ is lower than that of silicon $(\sim 4.5 \times$ $\left.10^{-18} \mathrm{~m}^{2} / \mathrm{W}\right)$, while its negligible nonlinear loss even up to extremely high light intensities yields a nonlinear figure of merit $(\gg>1)$ that is much higher than that of silicon ( 0.3) [5]. Figure 6b shows microscopic images of an integrated MRR patterned with 50 layers of GO $(\sim 50 \mu \mathrm{m}$ pattern length). Note that only the center ring of the 9 concentric rings (see inset) was coupled to through/drop bus waveguides to form an MRR - the rest were to aid in visual identification. Figure 6c shows the measured Raman spectra of an integrated chip (including doped silica MRRs) without GO and with 2 layers of uniformly coated GO film. The presence of the representative D $(1345 \mathrm{~cm}-1)$ and G $(1590 \mathrm{~cm}-1)$ peaks of GO confirms the integration of GO film onto the top surface. 

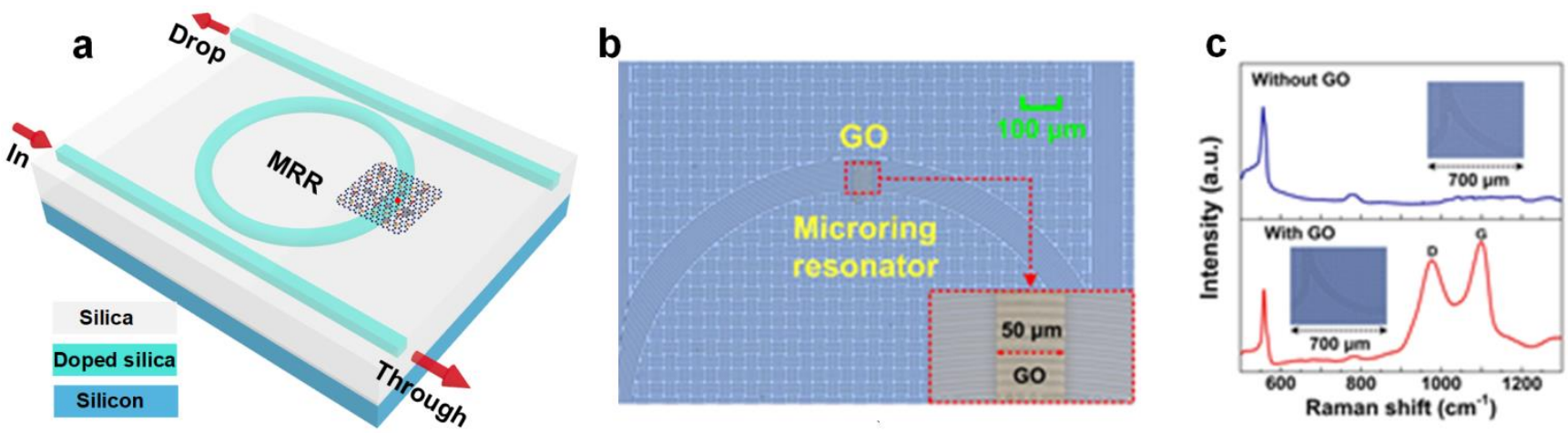

Figure 6. (a) Schematic illustration of GO-coated integrated MRR. Inset shows schematic atomic structure of GO. (b) Microscopic image of an integrated MRR patterned with 50 layers of GO. Inset shows zoom-in view of the patterned GO film. (c) Raman spectra of an integrated chip without GO and with 2 layers of GO. Insets show the corresponding microscope images.

Figure 7a shows the FWM spectra of the MRRs uniformly coated with 1-5 layers of GO, together with the FWM spectrum of the uncoated MRR. For comparison, we kept the same pump power of $\sim 22 \mathrm{dBm}$ coupled into the MRRs after excluding the mode coupling loss between the SMF array and the input bus waveguide as well as the GO-induced propagation loss of the input bus waveguide. The pump and signal had the same power and were separated in wavelength by 2 FSRs of the MRRs. As compared with the uncoated MRR, the GO-coated MRRs had an additional insertion loss (defined as the excess insertion loss of the GO-coated MRRs over the uncoated MRR), while the MRRs with 1 and 2 layers of GO clearly show enhanced idler output powers. The $\mathrm{CE}$ of the MRR without GO and with 1 layer of GO were $\sim 48.4 \mathrm{~dB}$ and $\sim 40.8 \mathrm{~dB}$, respectively, corresponding to a CE enhancement of $7.6 \mathrm{~dB}$ for the GO-coated MRR. Figure $7 \mathrm{~b}$ shows the FWM spectra of the MRRs with 10-50 layers of patterned GO. The GO coating length was $\sim 50 \mu \mathrm{m}$ and the input pump power ( $22 \mathrm{dBm}$ ) was the same as that in Figure 7a. The MRRs with patterned GO films also had an additional insertion loss as compared with the uncoated MRR, and the results for all the tested GO layer numbers show enhanced idler output powers. In particular, there is a maximum CE enhancement of $\sim 10.3 \mathrm{~dB}$ for the MRR patterned with 50 layers of GO. The CE enhancement and additional insertion loss extracted from Figures $7 \mathrm{a}$ and $\mathrm{b}$ are shown in Figures $7 \mathrm{c}$ and $\mathrm{d}$, respectively. As can be seen, the additional insertion loss increases with the GO layer number. For the MRRs with uniformly coated GO, the CE enhancement decreases with the GO layer number, whereas the MRRs with patterned GO shows the opposite trend. This could reflect the trade-off between FWM enhancement (which dominates for the patterned MRRs with a short GO coating length) and loss (which dominates for the uniformly coated MRRs with a much longer GO coating length) in the GO-coated MRRs.
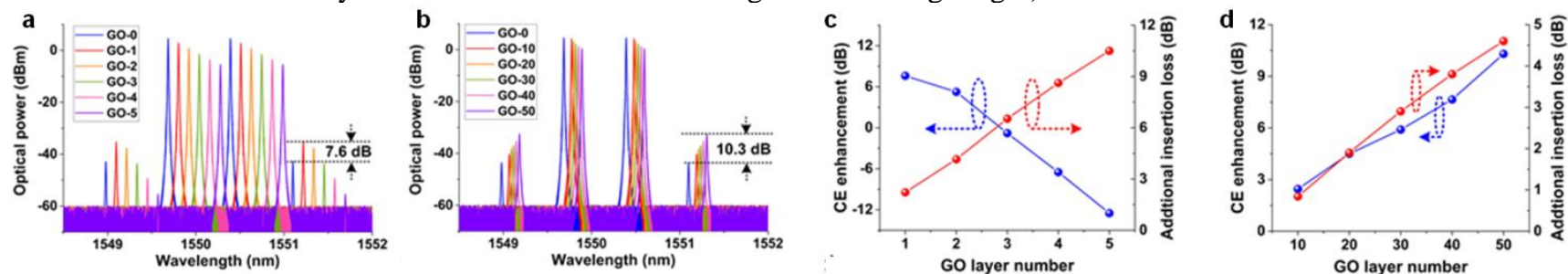

Figure 7. (a)-(b) Optical spectra of FWM at a pump power of $22 \mathrm{dBm}$ for the MRRs with 1-5 layers of uniformly coated and 10-50 layers of patterned GO films, respectively. (c)-(d) CE enhancement and additional insertion loss extracted from (a) and (b), respectively. In (a) and (b), the results for uncoated MRR (GO-0) are also shown for comparison.

\section{CONCLUSION}

We demonstrate enhanced nonlinear optics including Kerr nonlinearity and FWM in nanowires, waveguides, and ring resonators incorporated with layered GO films. We achieve precise control of the placement, thickness, and length of the GO films using layer-by-layer coating of GO films followed by photolithography and lift-off. Owing to the strong mode overlap between the platforms and the highly nonlinear GO films, we achieve a high nonlinear parameter of GO coated SOI nanowires up to 16 times and an improved nonlinear FOM of up to a factor of 20. Also, we obtain a significant improvement 
in the FWM CE of $\approx 6.1 \mathrm{~dB}$ for a uniformly coated Hydex waveguide with 2 layer of GO and $\approx 9.1 \mathrm{~dB}$ for a patterned SiN waveguide with 5 layers of GO. For MRRs, we achieve up to $\sim 10.3 \mathrm{~dB}$ in the FWM CE. Further recent theoretical work has shown that these results can be significantly improved, [42-47] aided by sophisticated integration methods. [48] This work will potentially have significant implications for on-chip microcombs and their applications. [49-169] In summary, our results verify the enhanced nonlinear optical performance of nanowires, waveguides, and ring resonators achievable by incorporating 2D layered GO films.

\section{REFERENCES}

[1] S. T. Cundiff, "Metrology - New generation of combs," Nature, vol. 450, no. 7173, pp. 1175-1176, Dec 20, 2007.

[2] A. Pasquazi, M. Peccianti, L. Razzari, D. J. Moss, S. Coen, M. Erkintalo, Y. K. Chembo, T. Hansson, S. Wabnitz, P. Del'Haye, X. Xue, A. M. Weiner, and R. Morandotti, "Micro-combs: A novel generation of optical sources," Physics Reports, vol. 729, pp. 1-81, 2018.

[3] H. Guo, C. Herkommer, A. Billat, D. Grassani, C. Zhang, M. H. P. Pfeiffer, W. Weng, C.-S. Brès, and T. J. Kippenberg, "Mid-infrared frequency comb via coherent dispersive wave generation in silicon nitride nanophotonic waveguides," Nature Photonics, vol. 12, no. 6, pp. 330-335, 2018.

[4] E. A. Kittlaus, H. Shin, and P. T. Rakich, "Large Brillouin amplification in silicon," Nature Photonics, vol. 10, no. 7, pp. 463-467, 2016.

[5] D. J. Moss, R. Morandotti, A. L. Gaeta, and M. Lipson, "New CMOS-compatible platforms based on silicon nitride and Hydex for nonlinear optics," Nature Photonics, vol. 7, no. 8, pp. 597-607, 2013/08/01, 2013.

[6] J. Leuthold, C. Koos, and W. Freude, "Nonlinear silicon photonics," Nature Photonics, vol. 4, no. 8, pp. 535-544, 2010/08/01, 2010.

[7] T. J. Kippenberg, A. L. Gaeta, M. Lipson, and M. L. Gorodetsky, "Dissipative Kerr solitons in optical microresonators," Science, vol. 361, no. 6402, pp. 567-+, Aug 10, 2018.

[8] M. Ferrera, L. Razzari, D. Duchesne, R. Morandotti, Z. Yang, M. Liscidini, J. E. Sipe, S. Chu, B. E. Little, and D. J. Moss, "Low-power continuous-wave nonlinear optics in doped silica glass integrated waveguide structures," Nature Photonics, vol. 2, no. 12, pp. 737-740, 2008/12/01, 2008.

[9] L. Razzari, D. Duchesne, M. Ferrera, R. Morandotti, S. Chu, B. E. Little, and D. J. Moss, "CMOS-compatible integrated optical hyper-parametric oscillator," Nature Photonics, vol. 4, no. 1, pp. 41-45, 2010/01/01, 2010.

[10] M. H. P. Pfeiffer, A. Kordts, V. Brasch, M. Zervas, M. Geiselmann, J. D. Jost, and T. J. Kippenberg, "Photonic Damascene process for integrated high-Q microresonator based nonlinear photonics," Optica, vol. 3, no. 1, pp. 2025, 2016/01/20, 2016.

[11] J. S. Levy, A. Gondarenko, M. A. Foster, A. C. Turner-Foster, A. L. Gaeta, and M. Lipson, "CMOS-compatible multiple-wavelength oscillator for on-chip optical interconnects," Nature Photonics, vol. 4, no. 1, pp. 37-40, 2010/01/01, 2010.

[12] K. P. Loh, Q. Bao, G. Eda, and M. Chhowalla, "Graphene oxide as a chemically tunable platform for optical applications," Nature Chemistry, vol. 2, no. 12, pp. 1015-1024, 2010/12/01, 2010.

[13] X. Li, H. Ren, X. Chen, J. Liu, Q. Li, C. Li, G. Xue, J. Jia, L. Cao, A. Sahu, B. Hu, Y. Wang, G. Jin, and M. Gu, "Athermally photoreduced graphene oxides for three-dimensional holographic images," Nature Communications, vol. 6, no. 1, pp. 6984, 2015/04/22, 2015.

[14] X. Zheng, B. Jia, H. Lin, L. Qiu, D. Li, and M. Gu, "Highly efficient and ultra-broadband graphene oxide ultrathin lenses with three-dimensional subwavelength focusing," Nature Communications, vol. 6, no. 1, pp. 8433, 2015/09/22, 2015.

[15] H. Lin, B. C. P. Sturmberg, K.-T. Lin, Y. Yang, X. Zheng, T. K. Chong, C. M. de Sterke, and B. Jia, “A 90-nmthick graphene metamaterial for strong and extremely broadband absorption of unpolarized light," Nature Photonics, vol. 13, no. 4, pp. 270-276, 2019/04/01, 2019.

[16] X. Zheng, B. Jia, X. Chen, and M. Gu, "In Situ Third-Order Non-linear Responses During Laser Reduction of Graphene Oxide Thin Films Towards On-Chip Non-linear Photonic Devices," Advanced Materials, vol. 26, no. 17, pp. 2699-2703, 2014/05/01, 2014. 
[17] K.-T. Lin, H. Lin, T. Yang, and B. Jia, "Structured graphene metamaterial selective absorbers for high efficiency and omnidirectional solar thermal energy conversion," Nature Comm., vol. 11, no. 1, pp. 1389, 2020/03/13, 2020.

[18] J. Wu, L. Jia, Y. Zhang, Y. Qu, B. Jia, and D. J. Moss, "Graphene Oxide for Integrated Photonics and Flat Optics," Advanced Materials, Vol. 33 (3) 2006415 (2021). DOI:10.1002/adma.202006415.

[19] X. Xu, X. Zheng, F. He, Z. Wang, H. Subbaraman, Y. Wang, B. Jia, and R. T. Chen, "Observation of Third-order Nonlinearities in Graphene Oxide Film at Telecommunication Wavelengths," Scientific Reports, vol. 7, no. 1, pp. 9646, 2017/08/29, 2017.

[20] Y. Yang, H. Lin, B. Y. Zhang, Y. Zhang, X. Zheng, A. Yu, M. Hong, and B. Jia, "Graphene-Based Multilayered Metamaterials with Phototunable Architecture for on-Chip Photonic Devices," ACS Photonics, vol. 6, no. 4, pp. 1033-1040, 2019/04/17, 2019.

[21] Y. Yang, J. Wu, X. Xu, Y. Liang, S. T. Chu, B. E. Little, R. Morandotti, B. Jia, and D. J. Moss, "Invited Article: Enhanced four-wave mixing in waveguides integrated with graphene oxide," APL Photonics, vol. 3, no. 12, pp. 120803, 2018/12/01, 2018.

[22] J. Wu, Y. Yang, Y. Qu, L. Jia, Y. Zhang, X. Xu, S. T. Chu, B. E. Little, R. Morandotti, B. Jia, and D. J. Moss, "2D Layered Graphene Oxide Films Integrated with Micro-Ring Resonators for Enhanced Nonlinear Optics," Small, vol. 16, no. 16, pp. 1906563, 2020/04/01, 2020.

[23] Y. Zhang, J. Wu, Y. Yang, Y. Qu, L. Jia, T. Moein, B. Jia, and D. J. Moss, "Enhanced Kerr Nonlinearity and Nonlinear Figure of Merit in Silicon Nanowires Integrated with 2D Graphene Oxide Films," ACS Applied Materials \& Interfaces, vol. 12, no. 29, pp. 33094-33103, 2020/07/22, 2020.

[24] Y. Qu, J. Wu, Y. Yang, Y. Zhang, Y. Liang, H. El Dirani, R. Crochemore, P. Demongodin, C. Sciancalepore, C. Grillet, C. Monat, B. Jia, and D. J. Moss, "Enhanced Four-Wave Mixing in Silicon Nitride Waveguides Integrated with 2D Layered Graphene Oxide Films," Advanced Optical Materials, Vol. 8 (21) 2001048, 2020. DOI: 10.1002/adom.202001048.

[25] W. Bogaerts, and L. Chrostowski, "Silicon Photonics Circuit Design: Methods, Tools and Challenges," Laser \& Photonics Reviews, vol. 12, no. 4, pp. 1700237, 2018/04/01, 2018.

[26] A. Autere, H. Jussila, Y. Dai, Y. Wang, H. Lipsanen, and Z. Sun, "Nonlinear Optics with 2D Layered Materials," Advanced Materials, vol. 30, no. 24, pp. 1705963, 2018/06/01, 2018.

[27] J. Wu, Y. Yang, Y. Qu, X. Xu, Y. Liang, S. T. Chu, B. E. Little, R. Morandotti, B. Jia, and D. J. Moss, "Graphene Oxide Waveguide and Micro-Ring Resonator Polarizers," Laser \& Photonics Reviews, vol. 13, no. 9, pp. 1900056, 2019/09/01, 2019.

[28] L. Liu, K. Xu, X. Wan, J. Xu, C. Y. Wong, and H. K. Tsang, "Enhanced optical Kerr nonlinearity of MoS_2 on silicon waveguides," Photonics Research, vol. 3, no. 5, pp. 206, 2015.

[29] Q. Feng, H. Cong, B. Zhang, W. Wei, Y. Liang, S. Fang, T. Wang, and J. Zhang, "Enhanced optical Kerr nonlinearity of graphene/Si hybrid waveguide," Applied Physics Letters, vol. 114, no. 7, pp. 071104, 2019/02/18, 2019.

[30] G. Cao, H. Lin, S. Fraser, X. Zheng, B. Del Rosal, Z. Gan, S. Wei, X. Gan, and B. Jia, "Resilient graphene ultrathin flat lens in aerospace, chemical, and biological harsh environments," ACS applied materials \& interfaces, vol. 11, no. 22, pp. 20298-20303, 2019.

[31] P. Demongodin, H. El Dirani, J. Lhuillier, R. Crochemore, M. Kemiche, T. Wood, S. Callard, P. Rojo-Romeo, C. Sciancalepore, C. Grillet, and C. Monat, "Ultrafast saturable absorption dynamics in hybrid graphene/Si3N4 waveguides," APL Photonics, vol. 4, no. 7, pp. 076102, 2019/07/01, 2019.

[32] T. Gu, N. Petrone, J. F. McMillan, A. van der Zande, M. Yu, G. Q. Lo, D. L. Kwong, J. Hone, and C. W. Wong, "Regenerative oscillation and four-wave mixing in graphene optoelectronics," Nature Photonics, vol. 6, no. 8, pp. 554-559, 2012/08/01, 2012.

[33] M. Ji, H. Cai, L. Deng, Y. Huang, Q. Huang, J. Xia, Z. Li, J. Yu, and Y. Wang, "Enhanced parametric frequency conversion in a compact silicon-graphene microring resonator," Optics express, vol. 23, no. 14,18679-18685, 2015.

[34] X. Xu, J. Wu, T. G. Nguyen, M. Shoeiby, S. T. Chu, B. E. Little, R. Morandotti, A. Mitchell, and D. J. Moss, "Advanced RF and microwave functions based on an integrated optical frequency comb source," Optics Express, vol. 26, no. 3, pp. 2569-2583, 2018. 
[35] X. Xu, J. Wu, M. Shoeiby, T. G. Nguyen, S. T. Chu, B. E. Little, R. Morandotti, A. Mitchell, and D. J. Moss, "Reconfigurable broadband microwave photonic intensity differentiator based on an integrated optical frequency comb source," Apl Photonics, vol. 2, no. 9, pp. 096104, 2017.

[36] H. El Dirani, A. Kamel, M. Casale, S. Kerdiles, C. Monat, X. Letartre, M. Pu, L. K. Oxenløwe, K. Yvind, and C. Sciancalepore, "Annealing-free Si3N4 frequency combs for monolithic integration with Si photonics," Applied Physics Letters, vol. 113, no. 8, pp. 081102, 2018/08/20, 2018.

[37] P. Demongodin, H. El Dirani, J. Lhuillier, R. Crochemore, M. Kemiche, T. Wood, S. Callard, P. Rojo-Romeo, C. Sciancalepore, C. Grillet, and C. Monat, "Ultrafast saturable absorption dynamics in hybrid graphene/Si3N4 waveguides," APL Photonics, vol. 4, no. 7, Jul, 2019.

[38] M. A. Foster, A. C. Turner, J. E. Sharping, B. S. Schmidt, M. Lipson, and A. L. Gaeta, "Broad-band optical parametric gain on a silicon photonic chip," Nature, vol. 441, no. 7096, pp. 960-3, Jun 22, 2006.

[39] T. Jiang, D. Huang, J. Cheng, X. Fan, Z. Zhang, Y. Shan, Y. Yi, Y. Dai, L. Shi, K. Liu, C. Zeng, J. Zi, J. E. Sipe, Y.-R. Shen, W.-T. Liu, and S. Wu, "Gate-tunable third-order nonlinear optical response of massless Dirac fermions in graphene," Nature Photonics, vol. 12, no. 7, pp. 430-436, 2018/07/01, 2018.

[40] M. Kues, C. Reimer, P. Roztocki, L. R. Cortés, S. Sciara, B. Wetzel, Y. Zhang, A. Cino, S. T. Chu, and B. E. Little, "On-chip generation of high-dimensional entangled quantum states and their coherent control," Nature, vol. 546, no. 7660, pp. 622-626, 2017.

[41] C. Reimer, M. Kues, P. Roztocki, B. Wetzel, F. Grazioso, B. E. Little, S. T. Chu, T. Johnston, Y. Bromberg, and L. Caspani, "Generation of multiphoton entangled quantum states by means of integrated frequency combs," Science, vol. 351, no. 6278, pp. 1176-1180, 2016.

[42] Y. Zhang, J. Wu, Y. Qu, L. Jia, B. Jia, and D. J. Moss, "Design and optimization of four-wave mixing in microring resonators integrated with 2D graphene oxide films", Journal of Lightwave Technology Vol. 39, (20) 6553-6562 (2021). DOI:10.1109/JLT.2021.3101292.

[43] Y. Zhang, J. Wu, Y. Qu, L. Jia, B. Jia, and D. J. Moss, "Optimizing the Kerr nonlinear optical performance of silicon waveguides integrated with 2D graphene oxide films", Journal of Lightwave Technology Vol. 39, (14) 46714683 (2021). DOI: 10.1109/JLT.2021.3069733.

[44] Y. Qu, J. Wu, Y. Zhang, Y. Liang, B. Jia, and D. J. Moss, “Analysis of four-wave mixing in silicon nitride waveguides integrated with 2D layered graphene oxide films", Journal of Lightwave Technology Vol. 39 (9) 29022910 (2021). DOI: 10.1109/JLT.2021.3059721.

[45] Y. Zhang, Y. Qu, J. Wu, L. Jia, Y. Yang, X. Xu, B. Jia, and D. J. Moss, "Graphene oxide for enhanced nonlinear optics in silicon and other CMOS compatible platforms", Paper IW1A.1, Integrated Photonics Research, Silicon and Nanophotonics, OSA Advanced Photonics Congress, Montreal, Canada, 26 - 29 July (2021).

[46] Y. Zhang, J. Wu, Y. Yang, Y. Qu, L. Jia, Y. Liang, H. El Dirani, R. Crochemore, P. Demongodinc, C. Sciancalepore, C. Grillet, C. Monat,B. Jia, and D. J. Moss, "Enhanced Kerr nonlinear performance in graphene oxide-coated silicon and silicon nitride waveguides", Paper SF2O.7, Conf. for Lasers and Electro-Optics, San Jose CA, May 9 - 14 (2021).

[47] Y. Qu, J. Wu, Y. Zhang, L. Jia, Y. Yang, X. Xu, S. T. Chu, B. E. Little, R. Morandotti, B. Jia, and D. J. Moss, "Graphene oxide for enhanced optical nonlinear performance in CMOS compatible integrated devices", Paper 11688-30, PW21O-OE109-36, 2D Photonic Materials and Devices IV, SPIE Photonics West, San Francisco CA March 6 -11 (2021). doi.org/10.1117/12.2583978

[48] L. Jia, J. Wu, Y. Zhang, Y. Qu, B. Jia, Z. Chen, and D. J. Moss, "Fabrication Technologies for the On-Chip Integration of 2D Materials", Small: Methods, Vol. 6, 2101435 (2022). DOI:10.1002/smtd.202101435.

[49] X. Xu et al, "Photonic perceptron based on a Kerr microcomb for scalable high speed optical neural networks", Laser and Photonics Reviews, vol. 14, no. 8, 2000070 (2020). DOI: 10.1002/lpor.202000070.

[50] X. Xu, et al., "11 TOPs photonic convolutional accelerator for optical neural networks", Nature 589, 44-51 (2021).

[51] Feldmann, J. et al., "Parallel convolutional processing using an integrated photonic tensor core", Nature 589, 52-58 (2021).

[52] T. Zhou et al, "Large-scale neuromorphic optoelectronic computing with a reconfigurable diffractive processing unit", Nature Photonics Vol 15, (5) 367 (2021). 
[53] B. J. Shastri et al., "Photonics for artificial intelligence and neuromorphic computing", Nature Photonics 15, (2) 102-114 (2021).

[54] Tait, A. N. et al., "Demonstration of WDM weighted addition for principal component analysis", Optics Express 23, 1275812765 (2015).

[55] Pasquazi, A. et al. Micro-combs: a novel generation of optical sources. Physics Reports 729, 1-81 (2018).

[56] Moss, D. J. et al., "New CMOS-compatible platforms based on silicon nitride and Hydex for nonlinear optics", Nature photonics 7, 597 (2013).

[57] Kippenberg, T. J., Gaeta, A. L., Lipson, M. \& Gorodetsky, M. L. Dissipative Kerr solitons in optical microresonators. Science 361, 567 (2018).

[58] Savchenkov, A. A. et al. Tunable optical frequency comb with a crystalline whispering gallery mode resonator. Physics Review Letters 101, 093902 (2008).

[59] Spencer, D. T. et al. An optical-frequency synthesizer using integrated photonics. Nature 557, 81-85 (2018).

[60] Marin-Palomo, P. et al. Microresonator-based solitons for massively parallel coherent optical communications. Nature 546, 274 (2017).

[61] B. Corcoran, et al., "Ultra-dense optical data transmission over standard fiber with a single chip source", Nature Communications, vol. 11, Article:2568, 2020.

[62] Kues, M. et al. Quantum optical microcombs. Nature Photonics 13, (3) 170-179 (2019). doi:10.1038/s41566-019-0363-0

[63] C. Reimer, L. Caspani, M. Clerici, et al., "Integrated frequency comb source of heralded single photons," Optics Express, vol. 22, no. 6, pp. 6535-6546, 2014.

[64] C. Reimer, et al., "Cross-polarized photon-pair generation and bi-chromatically pumped optical parametric oscillation on a chip", Nature Communications, vol. 6, Article 8236, 2015. DOI: 10.1038/ncomms9236.

[65] L. Caspani, C. Reimer, M. Kues, et al., "Multifrequency sources of quantum correlated photon pairs on-chip: a path toward integrated Quantum Frequency Combs," Nanophotonics, vol. 5, no. 2, pp. 351-362, 2016.

[66] C. Reimer et al., "Generation of multiphoton entangled quantum states by means of integrated frequency combs," Science, vol. 351, no. 6278, pp. 1176-1180, 2016.

[67] M. Kues, et al., "On-chip generation of high-dimensional entangled quantum states and their coherent control", Nature, vol. 546, no. 7660, pp. 622-626, 2017.

[68] P. Roztocki et al., "Practical system for the generation of pulsed quantum frequency combs," Optics Express, vol. 25, no. 16, pp. 18940-18949, 2017.

[69] Y. Zhang, et al., "Induced photon correlations through superposition of two four-wave mixing processes in integrated cavities", Laser and Photonics Reviews, vol. 14, no. 7, pp. 2000128, 2020. DOI: 10.1002/lpor.202000128

[70] C. Reimer, et al.,"High-dimensional one-way quantum processing implemented on d-level cluster states", Nature Physics, vol. 15, no.2, pp. 148-153, 2019.

[71] Stern, B., Ji, X., Okawachi, Y., Gaeta, A. L. \& Lipson, M. Battery-operated integrated frequency comb generator. Nature 562, 401 (2018).

[72] H. Bao, et al., Laser cavity-soliton microcombs, Nature Photonics, vol. 13, no. 6, pp. 384-389, Jun. 2019.

[73] Lugiato, L. A., Prati, F. \& Brambilla, M. Nonlinear Optical Systems, (Cambridge University Press, 2015).

[74] Cole, D. C., Lamb, E. S., Del'Haye, P., Diddams, S. A. \& Papp, S. B. Soliton crystals in Kerr resonators. Nature Photonics 11, 671 (2017).

[75] Wang, W., et al.., Robust soliton crystals in a thermally controlled microresonator, Opt. Lett., 43, 2002 (2018).

[76] Bao, C., et al., Direct soliton generation in microresonators, Opt. Lett, 42, 2519 (2017).

[77] M.Ferrera et al., "CMOS compatible integrated all-optical RF spectrum analyzer", Optics Express, vol. 22, no. 18, 21488 - $21498(2014)$.

[78] A. Pasquazi, et al., "Sub-picosecond phase-sensitive optical pulse characterization on a chip", Nature Photonics, vol. 5, no. 10, pp. 618-623 (2011).

[79] M. Kues, et al., "Passively modelocked laser with an ultra-narrow spectral width", Nature Photonics, vol. 11, no. 3, pp. 159, 2017.

[80] L. Razzari, et al., "CMOS-compatible integrated optical hyper-parametric oscillator," Nature Photonics, vol. 4, no. 1, pp. 41-45, 2010. 
[81] M. Ferrera, et al., "Low-power continuous-wave nonlinear optics in doped silica glass integrated waveguide structures," Nature Photonics, vol. 2, no. 12, pp. 737-740, 2008.

[82] M.Ferrera et al.“On-Chip ultra-fast $1^{\text {st }}$ and $2^{\text {nd }}$ order CMOS compatible all-optical integration”, Opt. Express, vol. 19, (23)pp. 23153-23161 (2011).

[83] D. Duchesne, M. Peccianti, M. R. E. Lamont, et al., "Supercontinuum generation in a high index doped silica glass spiral waveguide," Optics Express, vol. 18, no, 2, pp. 923-930, 2010.

[84] H Bao, L Olivieri, M Rowley, ST Chu, BE Little, R Morandotti, DJ Moss, ... "Turing patterns in a fiber laser with a nested microresonator: Robust and controllable microcomb generation”, Physical Review Research 2 (2), 023395 (2020).

[85] M. Ferrera, et al., "On-chip CMOS-compatible all-optical integrator", Nature Communications, vol. 1, Article 29, 2010.

[86] A. Pasquazi, et al., "All-optical wavelength conversion in an integrated ring resonator," Optics Express, vol. 18, no. 4, pp. 3858-3863, 2010.

[87] A. Pasquazi, Y. Park, J. Azana, et al., "Efficient wavelength conversion and net parametric gain via Four Wave Mixing in a high index doped silica waveguide," Optics Express, vol. 18, no. 8, pp. 7634-7641, 2010.

[88] M. Peccianti, M. Ferrera, L. Razzari, et al., "Subpicosecond optical pulse compression via an integrated nonlinear chirper," Optics Express, vol. 18, no. 8, pp. 7625-7633, 2010.

[89] Little, B. E. et al., "Very high-order microring resonator filters for WDM applications", IEEE Photonics Technol. Lett. 16, 2263-2265 (2004).

[90] M. Ferrera et al., "Low Power CW Parametric Mixing in a Low Dispersion High Index Doped Silica Glass MicroRing Resonator with Q-factor > 1 Million”, Optics Express, vol.17, no. 16, pp. 14098-14103 (2009).

[91] M. Peccianti, et al., "Demonstration of an ultrafast nonlinear microcavity modelocked laser", Nature Communications, vol. 3, pp. 765, 2012.

[92] A. Pasquazi, et al., "Self-locked optical parametric oscillation in a CMOS compatible microring resonator: a route to robust optical frequency comb generation on a chip," Optics Express, vol. 21, no. 11, pp. 13333-13341, 2013.

[93] A. Pasquazi, et al., "Stable, dual mode, high repetition rate mode-locked laser based on a microring resonator," Optics Express, vol. 20, no. 24, pp. 27355-27362, 2012.

[94] Wu, J. et al. RF Photonics: An Optical Microcombs' Perspective. IEEE Journal of Selected Topics in Quantum Electronics Vol. 24, 6101020, 1-20 (2018).

[95] Xu, X., et al., Photonic microwave true time delays for phased array antennas using a $49 \mathrm{GHz}$ FSR integrated microcomb source, Photonics Research, 6, B30-B36 (2018).

[96] T. G. Nguyen et al., "Integrated frequency comb source-based Hilbert transformer for wideband microwave photonic phase analysis," Opt. Express, vol. 23, no. 17, pp. 22087-22097, Aug. 2015.

[97] X. Xu, J. Wu, M. Shoeiby, T. G. Nguyen, S. T. Chu, B. E. Little, R. Morandotti, A. Mitchell, and D. J. Moss, "Reconfigurable broadband microwave photonic intensity differentiator based on an integrated optical frequency comb source," APL Photonics, vol. 2, no. 9, 096104, Sep. 2017.

[98] X. Xu, M. Tan, J. Wu, R. Morandotti, A. Mitchell, and D. J. Moss, "Microcomb-based photonic RF signal processing", IEEE Photonics Technology Letters, vol. 31 no. 23 1854-1857, 2019.

[99] X. Xu, et al., "Broadband RF channelizer based on an integrated optical frequency Kerr comb source," Journal of Lightwave Technology, vol. 36, no. 19, pp. 4519-4526, 2018.

[100] X. Xu, et al., "Continuously tunable orthogonally polarized RF optical single sideband generator based on micro-ring resonators," Journal of Optics, vol. 20, no. 11, 115701. 2018.

[101] X. Xu, et al., "Orthogonally polarized RF optical single sideband generation and dual-channel equalization based on an integrated microring resonator," Journal of Lightwave Technology, vol. 36, no. 20, pp. 4808-4818. 2018.

[102] M.Tan, X. Xu, J. Wu, B. Corcoran, A. Boes, T. G. Nguyen, Sai T. Chu, B. E. Little, R.Morandotti, A. Mitchell, and D. J. Moss, "Integral order photonic RF signal processors based on a soliton crystal micro-comb source", IOP Journal of Optics vol. 23 (11) 125701 (2021).

[103] X. Xu, et al., "Advanced adaptive photonic RF filters with 80 taps based on an integrated optical micro-comb source," Journal of Lightwave Technology, vol. 37, no. 4, pp. 1288-1295, 2019. 
[104] X. Xu, et al., Broadband microwave frequency conversion based on an integrated optical micro-comb source", Journal of Lightwave Technology, vol. 38 no. 2, pp. 332-338, 2020.

[105] M. Tan, et al., "Photonic RF and microwave filters based on 49GHz and 200GHz Kerr microcombs", Optics Comm. vol. 465,125563, Feb. 22. 2020.

[106] X. Xu, et al., "Broadband photonic RF channelizer with 90 channels based on a soliton crystal microcomb", Journal of Lightwave Technology, Vol. 38, no. 18, pp. 5116 - 5121, 2020. doi: 10.1109/JLT.2020.2997699.

[107] X. Xu, et al., "Photonic RF and microwave integrator with soliton crystal microcombs", IEEE Transactions on Circuits and Systems II: Express Briefs, vol. 67, no. 12, pp. 3582-3586, 2020. DOI:10.1109/TCSII.2020.2995682.

[108] X. Xu, et al., "Photonic RF phase-encoded signal generation with a microcomb source", J. Lightwave Technology, vol. 38 , no. 7, 1722-1727, 2020.

[109] X. Xu, et al., "High performance RF filters via bandwidth scaling with Kerr micro-combs," APL Photonics, vol. 4, no. 2, pp. 026102. 2019.

[110] M. Tan, et al., "Microwave and RF photonic fractional Hilbert transformer based on a $50 \mathrm{GHz}$ Kerr micro-comb", Journal of Lightwave Technology, vol. 37, no. 24, pp. 6097 - 6104, 2019.

[111] M. Tan, et al., "RF and microwave fractional differentiator based on photonics", IEEE Transactions on Circuits and Systems: Express Briefs, vol. 67, no.11, pp. 2767-2771, 2020. DOI:10.1109/TCSII.2020.2965158.

[112] M. Tan, et al., "Photonic RF arbitrary waveform generator based on a soliton crystal micro-comb source", Journal of Lightwave Technology, vol. 38, no. 22, pp. 6221-6226, Oct 22. 2020. DOI: 10.1109/JLT.2020.3009655.

[113] M. Tan, X. Xu, J. Wu, R. Morandotti, A. Mitchell, and D. J. Moss, "RF and microwave high bandwidth signal processing based on Kerr Micro-combs", Advances in Physics X, VOL. 6, NO. 1, 1838946 (2021). DOI:10.1080/23746149.2020.1838946.

[114] X. Xu, et al., "Advanced RF and microwave functions based on an integrated optical frequency comb source," Opt. Express, vol. 26 (3) 25692018.

[115] Kues, M. et al. Quantum optical microcombs. Nature Photonics 13, (3) 170-179 (2019). doi:10.1038/s41566-019-0363-0

[116] P.Roztocki et al., "Complex quantum state generation and coherent control based on integrated frequency combs", Journal of Lightwave Technology $\mathbf{3 7}$ (2) 338-347 (2019).

[117] S. Sciara et al., "Generation and Processing of Complex Photon States with Quantum Frequency Combs", IEEE Photonics Technology Letters 31 (23) 1862-1865 (2019). DOI: 10.1109/LPT.2019.2944564.

[118] L. Caspani, C. Reimer, M. Kues, et al., "Multifrequency sources of quantum correlated photon pairs on-chip: a path toward integrated Quantum Frequency Combs," Nanophotonics, vol. 5, no. 2, pp. 351-362, 2016.

[119] C. Reimer et al., "Generation of multiphoton entangled quantum states by means of integrated frequency combs," Science, vol. 351, no. 6278, pp. 1176-1180, 2016.

[120] M. Kues, et al., "On-chip generation of high-dimensional entangled quantum states and their coherent control", Nature, vol. 546, no. 7660, pp. 622-626, 2017.

[121] P. Roztocki et al., "Practical system for the generation of pulsed quantum frequency combs," Optics Express, vol. 25, no.16, 18940-18949, 2017.

[122] Y. Zhang, et al., "Induced photon correlations through superposition of two four-wave mixing processes in integrated cavities", Laser and Photonics Reviews, vol. 14, no. 7, pp. 2000128, 2020. DOI: 10.1002/lpor.202000128

[123] C. Reimer, et al.,"High-dimensional one-way quantum processing implemented on d-level cluster states", Nature Physics, vol. 15 (2) 148 (2019).

[124] H. Bao, et al., Laser cavity-soliton microcombs, Nature Photonics, vol. 13, no. 6, pp. 384-389, Jun. 2019.

[125] Bao, C., et al., Direct soliton generation in microresonators, Opt. Lett, 42, 2519 (2017).

[126] M. Ferrera et al., "CMOS compatible integrated all-optical RF spectrum analyzer", Optics Express, vol. 22, (18) 21488 (2014).

[127] A. Pasquazi, et al., "Sub-picosecond phase-sensitive optical pulse characterization on a chip", Nature Photonics, vol. 5, no. 10, pp. 618-623 (2011).

[128] M. Kues, et al., "Passively modelocked laser with an ultra-narrow spectral width", Nature Photonics, vol. 11, no. 3, pp. 159, 2017.

[129] L. Razzari, et al., "CMOS-compatible integrated optical hyper-parametric oscillator," Nature Photonics, vol. 4, no. $1,41-45,2010$. 
[130] M. Ferrera, et al., "Low-power continuous-wave nonlinear optics in doped silica glass integrated waveguide structures," Nature Photonics, vol. 2, no. 12, pp. 737-740, 2008.

[131] M.Ferrera et al."On-Chip ultra-fast $1^{\text {st }}$ and $2^{\text {nd }}$ order CMOS compatible all-optical integration”, Opt. Express, vol. 19, (23)pp. 23153-23161 (2011).

[132] D. Duchesne, M. Peccianti, M. R. E. Lamont, et al., "Supercontinuum generation in a high index doped silica glass spiral waveguide," Optics Express, vol. 18, no, 2, pp. 923-930, 2010.

[133] H Bao, L Olivieri, M Rowley, ST Chu, BE Little, R Morandotti, DJ Moss, ... "Turing patterns in a fiber laser with a nested microresonator: Robust and controllable microcomb generation”, Physical Review Research 2 (2), 023395 (2020).

[134] M. Ferrera, et al., "On-chip CMOS-compatible all-optical integrator", Nature Communications, vol. 1, Article 29, 2010.

[135] A. Pasquazi, et al., "All-optical wavelength conversion in an integrated ring resonator," Optics Express, vol. 18 (4) $3858(2010)$.

[136] A. Pasquazi, Y. Park, J. Azana, et al., "Efficient wavelength conversion and net parametric gain via Four Wave Mixing in a high index doped silica waveguide," Optics Express, vol. 18, no. 8, pp. 7634-7641, 2010.

[137] M. Peccianti, M. Ferrera, L. Razzari, et al., "Subpicosecond optical pulse compression via an integrated nonlinear chirper," Optics Express, vol. 18, no. 8, pp. 7625-7633, 2010.

[138] Little, B. E. et al., "Very high-order microring resonator filters for WDM applications", IEEE Phot. Technol. Lett. 16, 2263(2004).

[139] M. Ferrera et al., "Low Power CW Parametric Mixing in a Low Dispersion High Index Doped Silica Glass MicroRing Resonator with Q-factor > 1 Million”, Optics Express, vol.17, no. 16, pp. 14098-14103 (2009).

[140] M. Peccianti, et al., "Demonstration of an ultrafast nonlinear microcavity modelocked laser", Nature Comm., vol. 3, 765, 2012.

[141] A. Pasquazi, et al., "Self-locked optical parametric oscillation in a CMOS compatible microring resonator: a route to robust optical frequency comb generation on a chip," Optics Express, vol. 21, no. 11, pp. 13333-13341, 2013.

[142] A. Pasquazi, et al., "Stable, dual mode, high repetition rate mode-locked laser based on a microring resonator," Optics Express, vol. 20, no. 24, pp. 27355-27362, 2012.

[143] Xu, X., et al., Photonic microwave true time delays for phased array antennas using a $49 \mathrm{GHz}$ FSR integrated micro-comb source, Photonics Research, 6, B30-B36 (2018).

[144] X. Xu, M. Tan, J. Wu, R. Morandotti, A. Mitchell, and D. J. Moss, "Microcomb-based photonic RF signal processing”, IEEE Photonics Technology Letters, vol. 31 no. 23 1854-1857, 2019.

[145] M. Tan et al, "Orthogonally polarized Photonic Radio Frequency single sideband generation with integrated microring resonators", IOP Journal of Semiconductors, Vol. $\underline{42}$ (4), 041305 (2021). DOI: 10.1088/1674-4926/42/4/041305.

[146] Xu, et al., "Advanced adaptive photonic RF filters with 80 taps based on an integrated optical micro-comb source," Journal of Lightwave Technology, vol. 37, no. 4, pp. 1288-1295 (2019).

[147] X. Xu, et al., Broadband microwave frequency conversion based on an integrated optical micro-comb source", Journal of Lightwave Technology, vol. 38 no. 2, pp. 332-338, 2020.

[148] M. Tan, et al., "Photonic RF and microwave filters based on 49GHz and 200GHz Kerr microcombs", Optics Comm. vol. 465,125563, Feb. 22. 2020.

[149] X. Xu, et al., "Broadband photonic RF channelizer with 90 channels based on a soliton crystal microcomb", Journal of Lightwave Technology, Vol. 38, no. 18, pp. 5116 - 5121, 2020. doi: 10.1109/JLT.2020.2997699.

[150] X. Xu, et al., "Photonic RF and microwave integrator with soliton crystal microcombs", IEEE Transactions on Circuits and Systems II: Express Briefs, vol. 67, no. 12, pp. 3582-3586, 2020. DOI:10.1109/TCSII.2020.2995682.

[151] X. Xu, et al., "High performance RF filters via bandwidth scaling with Kerr micro-combs," APL Photonics, vol. 4 (2) 026102. 2019.

[152] M. Tan, et al., "Microwave and RF photonic fractional Hilbert transformer based on a $50 \mathrm{GHz}$ Kerr micro-comb", Journal of Lightwave Technology, vol. 37, no. 24, pp. 6097 - 6104, 2019.

[153] M. Tan, et al., "RF and microwave fractional differentiator based on photonics", IEEE Transactions on Circuits and Systems: Express Briefs, vol. 67, no.11, pp. 2767-2771, 2020. DOI:10.1109/TCSII.2020.2965158. 
[154] M. Tan, et al., "Photonic RF arbitrary waveform generator based on a soliton crystal micro-comb source", Journal of Lightwave Technology, vol. 38, no. 22, pp. 6221-6226 (2020). DOI: 10.1109/JLT.2020.3009655.

[155] M. Tan, X. Xu, J. Wu, R. Morandotti, A. Mitchell, and D. J. Moss, "RF and microwave high bandwidth signal processing based on Kerr Micro-combs", Advances in Physics X, VOL. 6, NO. 1, 1838946 (2021). DOI:10.1080/23746149.2020.1838946.

[156] X. Xu, et al., "Advanced RF and microwave functions based on an integrated optical frequency comb source," Opt. Express, vol. 26 (3) 2569 (2018).

[157] M. Tan, X. Xu, J. Wu, B. Corcoran, A. Boes, T. G. Nguyen, S. T. Chu, B. E. Little, R.Morandotti, A. Lowery, A. Mitchell, and D. J. Moss, ""Highly Versatile Broadband RF Photonic Fractional Hilbert Transformer Based on a Kerr Soliton Crystal Microcomb", Journal of Lightwave Technology vol. 39 (24) 7581-7587 (2021).

[158] Bao, C., et al., Direct soliton generation in microresonators, Opt. Lett, 42, 2519 (2017).

[159] Yuning Zhang, Yang Qu, Jiayang Wu, Linnan Jia, Yunyi Yang, Xingyuan Xu, Baohua Jia, and David J. Moss, "Enhanced Kerr nonlinearity and nonlinear figure of merit in silicon nanowires integrated with 2D graphene oxide films", ACS Applied Materials and Interfaces, Vol. 12 (29) 33094-33103 (2020). DOI:10.1021/acsami.0c07852

[160] D.Moss, "11 Tera-FLOP/s photonic convolutional accelerator and deep learning optical neural networks", Research Square, (2021). DOI: https://doi.org/10.21203/rs.3.rs-493347/v1.

[161] Moss, David (2020): “11.0 Tera-FLOP/second photonic convolutional accelerator for deep learning optical neural networks", TechRxiv. Preprint. (2020). https://doi.org/10.36227/techrxiv.13238423.v1

[162] Xu, X.; Tan, M.; Corcoran, B.; Wu, J.; Boes, A.; Nguyen, T.; Chu, S.; Little, B.; Hicks, D.; Morandotti, R.; Mitchell, A.; Moss, D. "11 Tera-FLOP per Second Photonic Convolutional Accelerator for Deep Learning Optical Neural Networks", Preprints 2020, 2020110420.

[163] Moss, David (2020): "RF and microwave photonic high bandwidth signal processing based on Kerr micro-comb sources", TechRxiv. (2020). Preprint. DOI:10.36227/techrxiv.12665609.v3

[164] Yuning Zhang, Jiayang Wu, Yunyi Yang, Yang Qu, Linnan Jia, Tania Moein, Baohua Jia, David J. Moss, "Enhanced nonlinear optical figure-of-merit at $1550 \mathrm{~nm}$ for silicon nanowires integrated with graphene oxide layered films", Arxiv (2020). arXiv:2004.08043 [physics.optics]

[165] Moss, David; Jia, Baohua; Wu, Jiayang; Zhang, Yuning; Yang, Yunyi; Jia, Linnan, Yang Qu, Tania Moein (2020): "Transforming silicon into a high performing integrated nonlinear photonics platform by integration with 2D graphene oxide films", TechRxiv. (2020). Preprint. DOI:10.36227/techrxiv.12061809.v1.

[166] A. Frigg, A. Boes, G. Ren, T.G. Nguyen, D.Y. Choi, S. Gees, D. Moss, A Mitchell, "Optical frequency comb generation with low temperature reactive sputtered silicon nitride waveguides", APL Photonics, Vol. 5 (1), 011302 (2020).

[167] T. Moein, D. Gailevičius, T. Katkus, S.H. Ng, S. Lundgaard, D.J. Moss, H. Kurt, Vygantas Mizeikis, Kęstutis Staliūnas, Mangirdas Malinauskas, Saulius Juodkazis, "Optically-thin broadband graphene-membrane photodetector", Nanomaterials, Vol. 10 (3), 407 (2020).

[168] M. Tan, X. Xu, J. Wu, A. Boes, B. Corcoran, T. G. Nguyen, S. T. Chu, B. E. Little, R. Morandotti, A. Mitchell, and D. J. Moss, "Advanced applications of Kerr mircocombs", Paper 11775-1. SPIE 11775, Integrated Optics: Design, Devices, Systems and Applications VI, (EOO21) OO107-8, Proc 1177504 (18 April 2021); Integrated Optics Conference, SPIE Optics and Optoelectronics Symposium, Prague, Czech Republic. April 19 - 22 (2021), doi.org/10.1117/12.2588733.

[169] Moss, David, "Microcombs for Ultrahigh Bandwidth Optical Data Transmission and Neural Networks." OSF Preprints. March 8. (2021). DOI:10.31219/osf.io/ne9wx. 